\title{
Risque de blessures à la tête et au visage chez les cyclistes en relation avec l'ajustement du casque : une étude cas-témoins
}

\author{
N. R. Romanow, M. Sc. (1); B. E. Hagel, Ph. D. (1, 2, 3); J. Williamson, B. H. Sc. (4); B. H. Rowe, M.D. (5)
}

Cet article a fait l'objet d'une évaluation par les pairs.

\section{Résumé}

Introduction : Nous avons examiné l'effet de l'ajustement et de la position du casque de vélo sur les blessures à la tête et au visage.

Méthodologie : Les cas étaient ceux de cyclistes portant un casque qui avaient subi des blessures à la tête $(n=297)$ ou au visage $(n=289)$. Les témoins étaient des cyclistes portant un casque qui avaient subi d'autres blessures, à l'exception des blessures au cou. Les participants ont été interrogés dans sept services d'urgence de l'Alberta ou par téléphone. Les données sur les blessures ont été recueillies au moyen des dossiers médicaux. Les valeurs manquantes ont été déduites à l'aide d'équations enchaînées et de modèles personnalisés d'imputation par prédiction.

Résultats : Comparativement aux sujets dont l'ajustement du casque était excellent, ceux dont l'ajustement était mauvais affichaient un plus grand risque de blessures à la tête (rapport de cotes $[\mathrm{RC}]=3,38$, intervalle de confiance [IC] à $95 \%: 1,06$ à 10,74). Comparativement aux sujets dont le casque était demeuré bien centré, ceux dont le casque s'était incliné vers l'arrière ( $\mathrm{RC}=2,90$, IC à $95 \%: 1,54$ à 5,47) ou s'était déplacé $(\mathrm{RC}=1,91$, IC à $95 \%: 1,01$ à 3,63) et ceux qui avaient perdu leur casque $(\mathrm{RC}=6,72$, IC à $95 \%: 2,86$ à 15,82) présentaient un plus grand risque de blessures à la tête. Un casque qui s'était incliné vers l'arrière $(\mathrm{RC}=4,81$, IC à $95 \%: 2,74$ à 8,46), s'était déplacé $(\mathrm{RC}=1,83$, IC à $95 \%: 1,04$ à 3,19) ou avait été perdu $(\mathrm{RC}=3,31$, IC à $95 \%$ : 1,24 à 8,85 ) augmentait aussi le risque de blessures au visage.

Conclusion : Nos observations ont des retombées sur les consommateurs et les programmes d'éducation des détaillants.

Mots-clés : dispositifs de protection de la tête, bicyclette, blessures

\section{Introduction}

Le casque de vélo réduit le risque de blessures à la tête et au visage lors d'un accident de vélo ${ }^{1}$. Cependant, bien des cyclistes ne portent pas leur casque correctement $^{2}$. La conception et la certification des casques de vélo se sont modifiées au cours des deux dernières décennies $^{3}$. Bien que le port obligatoire du casque soit de plus en plus répandu dans le monde, les lois qui le régissent varient d'un endroit à l'autre : certaines ne visent que les jeunes, alors que d'autres s'appliquent aux personnes de tout âge ${ }^{4,5}$. Des études comparatives dans des régions qui se sont dotées d'une loi sur le port du casque ont révélé une diminution globale du nombre de lésions traumatiques cérébrales déclarées ${ }^{4,6,7}$. Bien que ces études donnent du poids aux arguments en faveur d'une loi sur le port du casque, les efforts pour augmenter l'usage du casque pourraient ne pas se traduire par les bienfaits escomptés pour la santé si le casque est porté incorrectement.

Les tests de certification de la sûreté consistent habituellement en essais de chute, qui visent à s'assurer que l'impact se fait sentir bien au centre du casque, sur le dessus. Dans ce type d'essai, l'efficacité du casque est évaluée dans des conditions idéales, et le casque offre sa protection maximale lorsqu'il est correctement porté. Un ajustement correct est important dans les cas où le cycliste reçoit plusieurs coups à la tête. Si le casque reste en place après le premier coup, il protégera celui qui le porte contre les coups ultérieurs ${ }^{8}$.

La plupart des publications sur le port correct du casque de vélo traitent de la fréquence de l'usage correct $^{9}$, mais les rapports varient grandement en raison du manque d'uniformité des critères employés pour évaluer l'ajustement du casque. Une étude menée en 2010 a révélé que $20 \%$ des enfants de moins de 13 ans et $16,7 \%$ des enfants de 13 à 17 ans portaient mal leur casque ${ }^{10}$. L'erreur la plus fréquemment observée était un casque porté trop loin vers l'arrière de la tête. La bordure supérieure du casque protège contre les blessures au haut du visage en cas de collision frontale ${ }^{1,11}$, et le risque de ce type de blessure est significativement plus bas si le cycliste porte un casque ${ }^{1,12}$, bien qu'il semble nécessaire que le casque demeure en place pour offrir une protec-

Rattachement des auteurs :

1. Département de pédiatrie, Faculté de médecine, Université de Calgary, Alberta Children's Hospital, Calgary (Alberta), Canada

2. Département des sciences de la santé communautaire, Faculté de médecine, Université de Calgary, Alberta Children's Hospital, Calgary (Alberta), Canada

3. Alberta Children's Hospital Research Institute for Child and Maternal Health, Université de Calgary, Calgary (Alberta), Canada

4. Programme de baccalauréat en sciences de la santé, Faculté de médecine, Université de Calgary, Calgary (Alberta), Canada

5. Département de médecine d'urgence et École de santé publique, Université de l'Alberta, Edmonton (Alberta), Canada

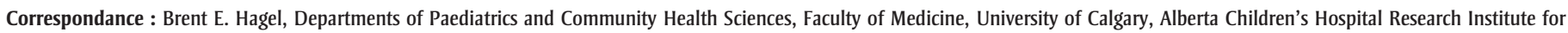
Child and Maternal Health, Alberta Children's Hospital, 2888 Shaganappi Trail NW, C4-434, Calgary (Alberta) T3B 6A8; tél. : 403-955-7403; téléc. : 403-955-3055;

courriel : brent.hagel@albertahealthservices.ca 
tion. Une seule étude a été menée pour déterminer la relation entre l'ajustement du casque de vélo et le risque de blessures à la tête ${ }^{13}$. Les auteurs ont estimé au double le risque de blessures à la tête avec un casque mal ajusté comparativement au risque avec un casque dont l'ajustement est excellent, au triple le risque lorsqu'il y a perte du casque pendant l'incident comparativement au risque avec un casque qui demeure bien centré, et à $52 \%$ de plus le risque lorsque le casque s'incline vers l'arrière comparativement au risque avec un casque qui demeure bien centré ${ }^{13}$. Bien que la méthodologie de cette étude soit adéquate, les données utilisées ont été recueillies il y a près de deux décennies, ce qui justifie d'étudier la question avec les casques de conception plus récente. De plus, aucune étude n'a évalué dans quelle mesure le port adéquat et l'ajustement correct du casque influent sur les blessures au visage chez les cyclistes.

Le but de notre étude était de déterminer la relation entre le risque de blessures à la tête ou au visage et les données autodéclarées concernant l'ajustement et la position du casque de vélo.

\section{Méthodologie}

\section{Collecte des données}

Les cyclistes blessés ont été recrutés dans sept services d'urgence de Calgary (Alberta Children's Hospital, Foothills Medical Centre, Rockyview General Hospital, Peter Lougheed Centre) et d'Edmonton (Stollery Children's Hospital, University of Alberta Hospital, Northeast Community Health Centre), en Alberta, sur une période de trois ans (de mai 2008 à octobre 2010). Nous avons identifié les cyclistes à l'aide du Regional Emergency Department Information System (système régional d'information des services d'urgence) et par un examen quotidien des dossiers des urgences, avec la collaboration du personnel des urgences. Les cyclistes blessés admissibles (ou les parents de ceux de moins de 14 ans) ont été abordés par le personnel de recherche ou, dans certains cas, par un médecin ou une infirmière des urgences, qui leur a demandé de participer à l'étude.
Après avoir donné leur consentement, les patients ont été interrogés aux urgences ou, s'ils ne souhaitaient pas répondre immédiatement, par téléphone. Si un patient admissible n'avait pas été vu aux urgences, le personnel de recherche lui faisait parvenir par la poste une trousse d'information sur l'étude avec un formulaire de consentement, et communiquait avec lui par téléphone pour lui demander d'y participer. Si un patient était hospitalisé après son séjour aux urgences, le personnel de recherche prenait les dispositions nécessaires pour l'informer au sujet de l'étude et l'interrogeait à l'hôpital s’il y consentait. Si le patient était trop jeune pour répondre aux questions, le personnel de recherche interrogeait un parent ou le tuteur. Si le parent ne connaissait pas les détails de l'accident ou ne pouvait pas répondre à une question, le personnel inscrivait " ne sait pas » ou " inconnu », selon le cas. Pour les entrevues par téléphone, la participation de l'enfant était demandée; cependant, si le parent refusait de laisser répondre l'enfant, les réponses du parent étaient acceptées.

Nous avons recueilli l'information sur les blessures à l'aide du dossier médical des patients. Nous avons exclu de l'étude les cyclistes blessés qui ne parlaient pas anglais, ceux qui n'avaient pas été vus aux urgences et qui n'avaient pas le téléphone ou qu'il a été impossible de joindre après un maximum de six appels, et ceux qui ont été blessés quand ils circulaient à l'intérieur ou utilisaient un vélo stationnaire. Nous n'avons pas non plus inclus les cyclistes blessés au cou, car la relation entre le port du casque et le risque de blessure au cou est moins claire ou moins admise ${ }^{7}$.

Dans le volet de l'étude axé sur l'ajustement du casque, nous avons établi deux groupes de cas distincts. Le premier se composait des cyclistes portant un casque qui avaient subi des blessures à la tête, quelle que soit la gravité de leurs autres blessures éventuelles. Une blessure à la tête était définie comme une blessure au cuir chevelu, au crâne ou au cerveau et ont été exclues les blessures aux vertèbres cervicales ou à la moelle épinière, les blessures au point de jonction du crâne et de la colonne vertébrale et celles au cou ou au visage. Les limites du crâne ont été définies comme une ligne imaginaire allant de la position normale des sourcils latéralement vers la lisière normale des cheveux, puis descendant vers l'arrière, sans les oreilles, et contournant la base de l'occiput.

Comme certaines données indiquent que le casque de vélo prévient les blessures au visage $^{1}$, notre deuxième groupe de cas se composait de cyclistes munis d'un casque qui avaient été blessés au visage, quelle que soit la gravité de leurs autres blessures éventuelles. Une blessure au visage était définie comme une blessure se situant entre la lisière normale des cheveux et le bas de la mandibule et, latéralement, d'une oreille à l'autre, oreilles comprises. Les cyclistes qui avaient à la fois des blessures à la tête et au visage ont été inclus dans les deux groupes. Les témoins, recrutés dans les mêmes services d'urgence que les cas à l'étude, étaient des cyclistes portant le casque qui avaient été blessés sous le niveau du cou et n'avaient aucune blessure à la tête, au cerveau ou au visage.

Nous avons interrogé les cyclistes aux urgences à l'aide d'un questionnaire structuré (disponible sur demande) basé sur des travaux antérieurs ${ }^{14,15}$ et mis à l'essai avec un échantillon de commodité. L'information recueillie portait à la fois sur le cycliste et sur les circonstances de l'accident. Pour l'analyse, nous avons mis l'accent sur l'information qui concernait le port et l'ajustement du casque. Les deux principales variables d'intérêt, l'ajustement du casque, d'une part, et sa position et son déplacement pendant l'accident, d'autre part, ont été autodéclarées à l'aide de réponses à choix multiples. En ce qui concerne l'ajustement du casque, les choix de réponse étaient les suivants : (1) excellent, (2) bon, (3) passable et (4) mauvais. Pour ce qui est de la position du casque, les choix de réponse étaient : (1) est demeuré bien centré, (2) s'est incliné vers l'arrière, (3) s'est déplacé vers le côté et (4) perte du casque. Pour les deux variables, les participants pouvaient aussi répondre "ne sais pas " ou "refuse de répondre », réponses qui ont toutes deux 
été considérées comme des données manquantes dans la première analyse.

Des entrevues de suivi ont été réalisées avec un sous-échantillon de patients afin d'établir la fiabilité de l'entrevue initiale. Le même questionnaire a été employé et le répondant initial a été prié de répondre au questionnaire de suivi (p. ex. le parent si c'est lui qui avait répondu la première fois). Les résultats des deux entrevues (la première et celle réalisée plus tard) ont été comparés à l'aide du coefficient kappa $(\kappa)^{16}$ avec un intervalle de confiance (IC) à $95 \%$. Les entrevues de suivi ont été réalisées au moins deux semaines après la première entrevue auprès des patients qui avaient accepté, au moment de la première entrevue, qu'on communique avec eux pour un suivi.

L'étude a été approuvée par le comité conjoint d'éthique de la recherche en santé de l'Université de Calgary et le comité d'éthique de la recherche en santé de l'Université de l'Alberta.

\section{Analyse des données}

Nous avons calculé les rapports de cotes (RC, avec IC à $95 \%$ ) bruts en ce qui concerne l'association entre l'ajustement du casque et les blessures à la tête ou au visage. Nous avons aussi examiné la relation entre la position du casque lors de l'accident et les blessures à la tête ou au visage. Des analyses de régression logistique multiple ont aussi été effectuées pour neutraliser l'effet des facteurs de confusion possibles (variables potentiellement liées à la position ou à l'ajustement du casque et facteurs de risque indépendants de blessures à la tête ou au visage), dont l'âge, le sexe, l'indice de masse corporelle (IMC), la fréquence d'utilisation de la bicyclette, la présence d'un compagnon de cyclisme et la vitesse estimative autodéclarée par le cycliste. Quatre catégories d'âge ont été établies : moins de 13 ans, 13 à 17 ans, 18 à 39 ans et 40 ans et plus. Les catégories d'IMC étaient basées sur la classification de l'Organisation mondiale de la Santé, soit l'insuffisance pondérale $\left(<18,50 \mathrm{~kg} / \mathrm{m}^{2}\right)$, le poids normal $\left(18,50\right.$ à $\left.24,99 \mathrm{~kg} / \mathrm{m}^{2}\right)$, le surpoids $\left(25,00\right.$ à $\left.29,99 \mathrm{~kg} / \mathrm{m}^{2}\right)$ et l'obésité $\left(\geq 30 \mathrm{~kg} / \mathrm{m}^{2}\right)^{17}$. La fréquence d'utilisation de la bicyclette était répartie entre les catégories suivantes : au moins une fois par semaine, au moins une fois par mois et au moins une fois par année ${ }^{18}$. On a noté si les cyclistes pédalaient seuls, avec des enfants, avec des adultes ou avec d'autres personnes (p. ex. chefs de camp). La vitesse déclarée a été scindée en deux catégories : moins de $25 \mathrm{~km} / \mathrm{h}$ et $25 \mathrm{~km} / \mathrm{h}$ et plus. Dans la méthode de modélisation par régression ascendante employée, chaque covariable a été ajoutée au modèle renfermant la variable étudiée (blessure à la tête ou au visage) et l'exposition (ajustement du casque ou position du casque) séparément. Des modèles distincts ont été conçus pour l'ajustement du casque et la position du casque afin d'éviter la colinéarité des deux variables. Lorsqu'une covariable produisait un changement égal ou supérieur à $10 \%$ dans les estimations relatives à l'ajustement ou à la position du casque, elle était retenue dans le modèle. Ce processus a été répété jusqu'à ce qu'aucun changement ne soit observé ou jusqu'à ce que le nombre de variables dans le modèle ait atteint $10 \%$ du nombre de cas ${ }^{19}$.

\section{Analyse à imputation multiple}

Nous avons déduit les valeurs manquantes concernant les variables d'exposition et les facteurs de confusion potentiels à l'aide d'équations enchaînées et de modèles personnalisés d'imputation par prédiction $^{20}$. Dans le modèle d'imputation, les variables ont été imputées selon la fréquence d'absence des valeurs (du moins grand nombre au plus grand nombre de valeurs manquantes) au moyen de la correspondance prédictive des moyennes (predictive mean matching) pour les variables continues et de la régression logistique multinomiale ou ordonnée pour les variables catégoriques lorsque nécessaire. Des prédicteurs non manquants ont aussi été inclus. Des modèles de régression logistique comprenant toutes les covariables (âge, sexe, IMC, vitesse du cycliste, fréquence d'utilisation de la bicyclette et compagnon de cyclisme) ont été utilisés pour estimer les $\mathrm{RC}$ et les IC à $95 \%$ à partir des données imputées. Toutes les analyses de données ont été réalisées à l'aide du logiciel STATA, version 11.0 (StataCorp LP, College Station, Texas, États-Unis).

\section{Résultats}

\section{Caractéristiques de l'échantillon}

Au total, 4960 cyclistes blessés se sont prêtés à une évaluation visant à déterminer leur admissibilité et 3111 (63\%) ont consenti à participer à l'étude et ont été recrutés. De ce nombre, 2336 (75 \%) portaient un casque au moment de l'accident. L'analyse a porté sur 297 cyclistes ayant subi des blessures à la tête, 289 cyclistes ayant subi des blessures au visage et 1694 témoins. Soixante-quatre participants avaient à la fois des blessures à la tête et au visage, et ils ont donc été inclus dans les deux groupes.

Le tableau 1 présente les caractéristiques des groupes de cyclistes ayant eu des blessures à la tête et au visage et les témoins. Si on les compare aux témoins, les cyclistes présentant des blessures à la tête roulaient généralement plus vite et plus souvent seuls, alors que ceux ayant des blessures au visage étaient plus jeunes, avaient un IMC plus bas, pédalaient seuls ou avec des adultes et portaient rarement un casque intégral.

\section{Ajustement et position du casque et risque de blessures à la tête}

D'après les estimations brutes, un mauvais ajustement du casque augmente significativement le risque de blessures à la tête par rapport à un ajustement excellent $(\mathrm{RC}=3,26$, IC à $95 \%: 1,08$ à 9,83$)$ (tableau 2). Lorsque le casque s'était incliné vers l'arrière $(\mathrm{RC}=2,76, \mathrm{IC}$ à $95 \%: 1,47$ à $5,18)$, s'était déplacé vers le côté ( $\mathrm{RC}=1,87$, IC à $95 \%: 1,03$ à 3,42) ou qu'il y avait eu perte du casque $(\mathrm{RC}=6,77$, IC à $95 \%: 3,08$ à 14,86), le risque de blessures à la tête augmentait significativement par rapport au groupe dont le casque était demeuré « bien centré ».

Les RC ajustés pour un ajustement bon, passable et mauvais du casque étaient de 0,96 (IC à $95 \%$ : 0,69 à 1,36), 1,93 (IC à $95 \%: 1,04$ à 3,57) et 3,23 (IC à $95 \%: 0,78$ à $13,41)$, respectivement, comparativement à un ajustement excellent. Chez les cyclistes qui avaient qualifié de passable l'ajustement de leur casque, le risque de blessures à la tête était près de deux fois plus élevé que 
TABLEAU 1

Caractéristiques de l'accident et des cyclistes, cas et témoins, qui ont été blessés à Calgary et à Edmonton (Alberta)

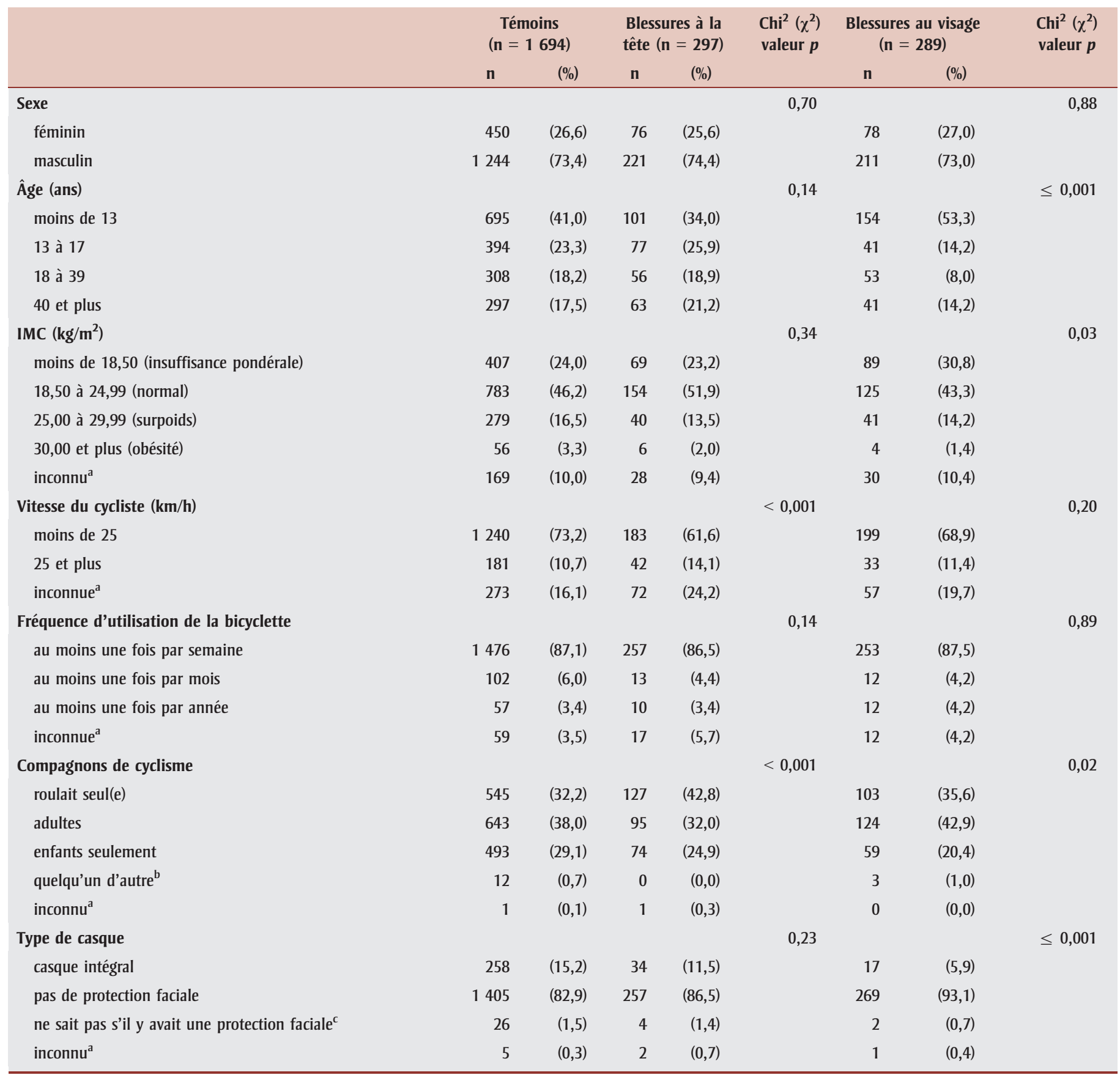

Abréviation: IMC, indice de masse corporelle.

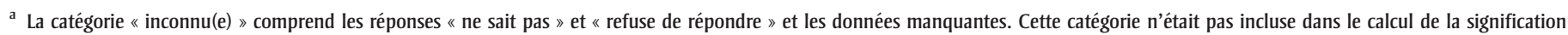
statistique.

b Comprend les réponses qu'il était impossible de classer parmi les compagnons « adultes » ou « enfants » (p. ex. vélo avec un instructeur ou une gardienne).

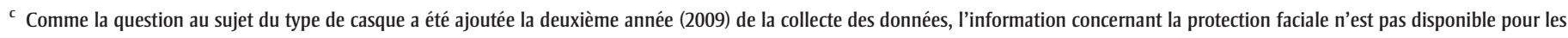
participants de la première année de l'étude (2008).

chez ceux dont l'ajustement était excellent. Après imputation, seuls ceux dont l'ajustement du casque avait été qualifié de mauvais $(\mathrm{RC}=3,38$, IC à $95 \%: 1,06$ à $10,74)$ affichaient un risque significative- ment élevé de blessures à la tête par rapport à ceux dont l'ajustement était excellent.

Après ajustement pour tenir compte des covariables, les cyclistes qui ont perdu leur casque pendant l'accident affichaient un risque de blessures à la tête sept fois plus élevé que ceux dont le casque était demeuré bien centré $(\mathrm{RC}=7,13$, IC à $95 \%: 2,94$ à 17,29$)$. Chez ceux dont le 
TABLEAU 2

Rapports de cotes estimés en ce qui concerne la relation entre l'ajustement du casque et les blessures à la tête parmi les cyclistes blessés à Calgary et à Edmonton (Alberta)

\begin{tabular}{|c|c|c|c|c|c|c|c|c|c|c|}
\hline & \multicolumn{2}{|c|}{$\begin{array}{l}\text { Témoins } \\
\text { (n=1 694) }\end{array}$} & \multicolumn{2}{|c|}{$\begin{array}{c}\text { Cas } \\
(n=297)\end{array}$} & \multicolumn{2}{|c|}{$\begin{array}{l}\text { RC brut } \\
\text { (IC à } 95 \%)\end{array}$} & \multicolumn{2}{|r|}{$\begin{array}{l}\text { RC ajusté } \\
\text { (IC à } 95 \%)\end{array}$} & \multicolumn{2}{|c|}{$\begin{array}{l}\text { RC ajusté imputéa } \\
\text { (IC à } 95 \% \text { ) }\end{array}$} \\
\hline & $\mathbf{n}$ & $(\%)$ & $\mathbf{n}$ & $(\%)$ & & & & & & \\
\hline \multicolumn{11}{|l|}{ Ajustement du casque $^{b}$} \\
\hline excellent & 1014 & $(59,9)$ & 173 & $(58,1)$ & 1,00 & (référence) & 1,00 & (référence) & 1,00 & (référence) \\
\hline bon & 579 & $(34,2)$ & 92 & $(30,9)$ & 0,93 & $(0,71$ à 1,22$)$ & 0,96 & $(0,69 \text { à } 1,36)^{c}$ & 0,97 & $(0,73$ à 1,29$)$ \\
\hline passable & 81 & $(4,8)$ & 22 & $(7,4)$ & 1,59 & $(0,97$ à 2,62$)$ & 1,93 & $(1,04 \text { à } 3,57)^{c}$ & 1,60 & $(0,96$ à 2,66$)$ \\
\hline \multicolumn{11}{|c|}{ Qu'est-il arrivé à votre casque? $^{d}$} \\
\hline est demeuré bien centré & 1421 & $(83,9)$ & 180 & $(60,4)$ & 1,00 & (référence) & 1,00 & (référence) & 1,00 & (référence) \\
\hline s'est incliné vers l'arrière & 40 & $(2,4)$ & 14 & $(4,7)$ & 2,76 & $(1,47$ à 5,18$)$ & 3,54 & $(1,70 \text { à } 7,40)^{e}$ & 2,90 & $(1,54$ à 5,47$)$ \\
\hline s'est déplacé vers le côté & 59 & $(3,5)$ & 14 & $(4,7)$ & 1,87 & $(1,03$ à 3,42$)$ & 1,84 & $(0,90 \text { à } 3,77)^{e}$ & 1,91 & $(1,01$ à 3,63$)$ \\
\hline perte du casque & 14 & $(0,8)$ & 12 & $(4,0)$ & 6,77 & $(3,08$ à 14,86$)$ & 7,13 & $(2,94 \text { à } 17,29)^{e}$ & 6,72 & $(2,86$ à 15,82$)$ \\
\hline
\end{tabular}

Abréviations : IC, intervalle de confiance; IMC, indice de masse corporelle; RC, rapport de cotes.

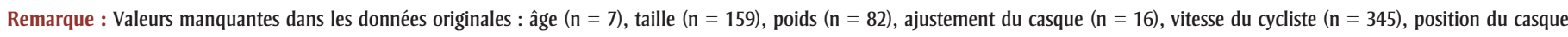
$(n=225)$, fréquence d'utilisation de la bicyclette $(n=76)$ et compagnon de cyclisme $(n=2)$.

a Estimations ajustées pour tenir compte de la fréquence d'utilisation de la bicyclette, de la présence d'un compagnon de vélo, de la vitesse, de l'ıMC, du sexe et de l'âge.

b L'analyse ajustée portait sur 198 cas et 1244 témoins avant imputation.

c Estimations ajustées pour tenir compte de la fréquence d'utilisation de la bicyclette, de la vitesse, de l'IMC et de l'âge.

d L'analyse ajustée portait sur 166 cas et 1178 témoins avant imputation.

e Estimations ajustées pour tenir compte de la vitesse, d'un compagnon de cyclisme et de I'IMC.

casque s'était incliné vers l'arrière, le risque de blessures à la tête était plus de trois fois supérieur ( $\mathrm{RC}=3,54$, IC à $95 \%$ : $1,70$ à 7,40$)$. Les estimations ajustées basées sur les données imputées étaient similaires : le RC estimé pour un casque qui s'était incliné vers l'arrière était de 2,90 (IC à $95 \%: 1,54$ à 5,47 ) et le RC estimé lorsqu'il y avait eu perte du casque était de 6,72 (IC à $95 \%: 2,86$ à 15,82). Le résultat dans le cas d'un casque qui s'était déplacé vers le côté était lui aussi significatif après imputation $(\mathrm{RC}=1,91$, IC à $95 \%: 1,01$ à $3,62)$.

\section{Ajustement et position du casque et risque de blessures au visage}

Les estimations brutes ont révélé un risque plus élevé de blessures au visage lorsque le casque s'était incliné vers l'arrière (RC = $4,19$, IC à $95 \%: 2,46$ à 7,15$)$, s'était déplacé vers le côté ( $\mathrm{RC}=1,98$, IC à $95 \%$ : $1,11$ à 3,50$)$ ou lorsqu'il y avait eu perte du casque $(\mathrm{RC}=3,12$, IC à $95 \%: 1,19$ à 8,22) (tableau 3 ). Cependant, après un ajustement pour tenir compte de l'IMC, de la fréquence d'utilisation de la bicyclette et de la vitesse du cycliste, seuls ceux dont le casque s'était incliné vers l'arrière présentaient un risque plus grand de blessures au visage ( $\mathrm{RC}=4,49$, IC à $95 \%: 2,30$ à 8,77). Un mauvais ajustement était un indicateur d'un effet néfaste, mais n'était pas statistiquement significatif (RC $=3,10$, IC à $95 \%$ : 0,76 à 12,69).

Comparativement aux estimations ajustées basées sur les données originales, les RC imputés pour ce qui est du risque de blessures au visage s'éloignaient de 1,00. Le risque de blessures au visage augmentait significativement lorsque le casque s'était incliné vers l'arrière ( $\mathrm{RC}=4,81$, IC à $95 \%: 2,74$ à 8,46), s'était déplacé vers le côté $(\mathrm{RC}=1,83$, IC à $95 \%: 1,04$ à $3,19)$ ou lorsqu'il y avait eu perte du casque $(\mathrm{RC}=3,31$, IC à $95 \%: 1,24$ à $8,85)$.

\section{Qualité et fiabilité des données}

En ce qui concerne l'ajustement du casque, la correspondance globale observée était de $87,5 \%$ et la correspondance attendue, de 81,0\% (tableau 4). La valeur du coefficient kappa pondéré a été calculée étant donné que les réponses étaient ordonnées dans le temps, et la valeur kappa était de 0,34 (IC à $95 \%$ : 0,16 à $0,64)$, ce qui représente une correspondance faible $\mathrm{e}^{14}$. Pour les cas de blessures à la tête et au visage $(n=24)$, la correspondance observée était de $91,7 \%$ et la correspondance attendue, de 79,8 \%, ce qui donne une valeur kappa de 0,59 (IC à $95 \%$ : 0,28 à 1,00), soit une correspondance moyenne. Dans le cas de témoins, la valeur kappa était de 0,22 (IC à $95 \%$ : $0,00$ à 0,44$)$.

Une valeur kappa non pondérée a été calculée pour ce qui est de la position du casque. Dans les cas de blessures à la tête et au visage, la concordance observée était de $62,5 \%$, la concordance attendue était de 49,3\% et la valeur kappa était de 0,26 (IC à $95 \%: 0,00$ à 0,54), ce qui représente une correspondance faible. Dans le cas de témoins, la concordance observée était de $90,6 \%$, la correspondance attendue, de $85,6 \%$ et la valeur kappa, de 0,35 (IC à $95 \%: 0,19$ à 0,71 ), ce qui représente une concordance faible. 
TABLEAU 3

Rapports de cotes estimés pour la relation entre l'ajustement du casque et les blessures au visage parmi les cyclistes blessés à Calgary et à Edmonton (Alberta)

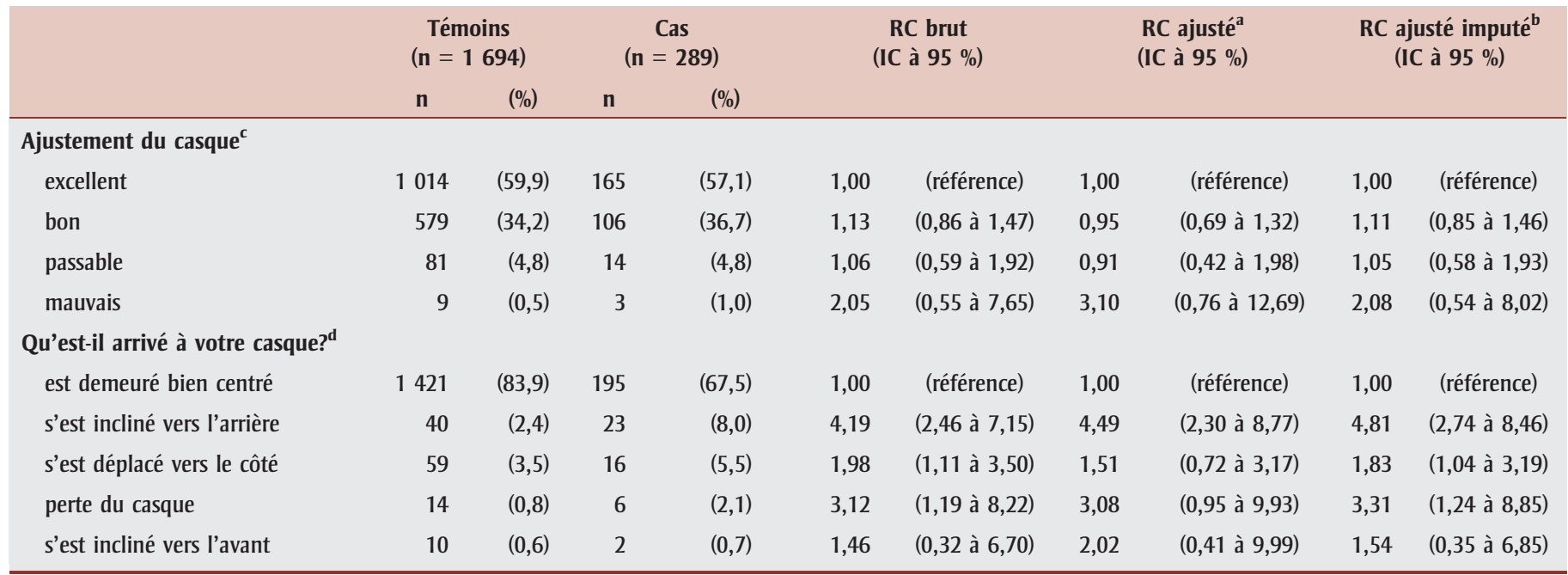

Abréviations : IC, intervalle de confiance; IMC, indice de masse corporelle; RC, rapport de cotes.

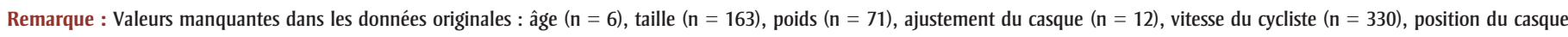
( $n=194)$, fréquence d'utilisation de la bicyclette $(n=71)$ et compagnon de cyclisme $(n=1)$.

${ }^{a}$ Estimations ajustées pour tenir compte de l'IMC, de la fréquence d'utilisation de la bicyclette et de la vitesse du cycliste.

b Estimations ajustées pour tenir compte de la fréquence d'utilisation de la bicyclette, d'un compagnon de cyclisme, de la vitesse, de l'ıMC, du sexe et de l'âge.

c L'analyse ajustée portait sur 198 cas et 1244 témoins avant imputation.

d L'analyse ajustée portait sur 170 cas et 1318 témoins avant imputation.

\section{Analyse}

L'étude fournit des données à jour sur la relation entre un ajustement correct du casque de vélo et le risque de blessures à la tête ou au visage. Bien que l'effet protecteur global du casque de vélo soit bien établi, de l'information précise sur l'ajustement et la position du casque nous permet de mieux comprendre les effets du casque et fournit des données que peuvent

utiliser les cyclistes, les fabricants de casques et les groupes qui s'occupent de la prévention des blessures.

Rivara et collab. ${ }^{13}$ ont fait état d'une hausse du risque de blessures à la tête lorsque le casque se déplace vers l'arrière ou qu'il y a perte du casque. Nos résultats étaient environ deux fois plus élevés que ceux signalés auparavant. Nous avons aussi observé une relation entre les

TABLEAU 4

Correspondance et valeur du coefficient kappa pour l'ajustement et la position du casque parmi les cyclistes (cas et témoins) blessés à Calgary et à Edmonton (Alberta)

\begin{tabular}{|c|c|c|c|c|}
\hline & $\begin{array}{c}\text { Correspondance } \\
\text { observée (\%) }\end{array}$ & $\begin{array}{l}\text { Correspondance } \\
\text { attendue (\%) }\end{array}$ & $\kappa$ & IC à 95 \% \\
\hline \multicolumn{5}{|l|}{ Cas $(n=24)$} \\
\hline Ajustement du casque & 91,7 & 79,8 & 0,59 & $(0,28$ à 1,00$)$ \\
\hline Position du casque & 62,5 & 49,3 & 0,26 & $(0,00$ à 0,54$)$ \\
\hline \multicolumn{5}{|l|}{ Témoins ( $n=53$ ) } \\
\hline Ajustement du casque & 85,5 & 81,6 & 0,22 & $(0,00$ à 0,44$)$ \\
\hline Position du casque & 90,6 & 85,6 & 0,35 & $(0,19$ à 0,71$)$ \\
\hline \multicolumn{5}{|l|}{ Total $(\mathbf{n}=\mathbf{7 7})$} \\
\hline Ajustement du casque & 87,5 & 81,0 & 0,34 & $(0,16$ à 0,64$)$ \\
\hline Position du casque & 81,8 & 72,0 & 0,35 & $(0,20$ à 0,74$)$ \\
\hline
\end{tabular}

Abréviations : IC, intervalle de confiance; $\kappa$, coefficient kappa. blessures à la tête et le déplacement du casque vers le côté, observation qui n’avait pas été faite auparavant. Nous n'avons pas constaté que l'ajustement autodéclaré du casque influait sur le risque de blessures au visage, mais ce risque augmentait par un facteur de trois lorsqu'il y avait eu perte du casque au moment de l'accident et de près de cinq fois lorsque le casque s'était déplacé vers l'arrière.

Foss et Beirness ${ }^{21}$ ont signalé que le port incorrect du casque était plus fréquent chez les 1 à 5 ans et les 6 à 15 ans que chez les cyclistes plus vieux et que le risque de blessures à la tête était plus grand chez les 6 à $15 \mathrm{ans}^{21}$. Selon leur définition, un casque mal porté était un casque dont la sangle n'était pas bouclée sous le menton ou qui était incliné vers l'arrière ${ }^{21}$. Nous avons aussi constaté que la proportion de blessures à la tête et au visage était élevée dans le groupe le plus jeune (moins de 13 ans) par rapport aux groupes plus vieux, phénomène qui pourrait s'expliquer par le déplacement du casque vers l'arrière au moment de l'accident. 
Dans une autre étude canadienne, les chercheurs ont observé que 4,3 \% des utilisateurs de casque le portaient incorrectement, soit incliné vers l'arrière, soit avec la sangle non bouclée sous le menton, soit par-dessus une casquette de baseball $^{22}$. Une étude d'observation menée en 2010 en Alberta $^{10}$ a révélé que 16,6 \% des cyclistes, dont $21 \%$ des enfants de moins de 13 ans, portaient mal leur casque. Dans notre étude, environ $9 \%$ des sujets ayant subi des blessures à la tête et $6 \%$ de ceux ayant subi des blessures au visage ont déclaré que l'ajustement de leur casque était passable ou mauvais comparativement à $5,3 \%$ des témoins. Ces chiffres sont probablement sous-estimés, car Lee et collab. ${ }^{9}$ ont constaté que la fréquence de l'usage correct du casque variait de $46 \%$ à $100 \%$ dans les études récentes, tout en notant un manque d'uniformité dans les définitions d'un usage correct.

Nos observations concernant l'importance de l'ajustement du casque permettent de mieux comprendre l'effet protecteur potentiel des casques de vélo. Les études précédentes ayant établi que le port du casque (par rapport à l'absence de casque) réduisait le risque de blessures à la tête ou au cerveau ${ }^{1}$ pourraient bien avoir sousestimé l'effet protecteur du casque étant donné qu'il est probable qu'un certain nombre de participants à ces études portaient un casque mal ajusté ou portaient leur casque incorrectement (p. ex. sangle non bouclée). Si c'est le cas, cela pourrait avoir une incidence sur les activités de promotion du port du casque, qui devraient être axées sur la bonne façon de porter le casque afin d'obtenir un effet protecteur maximal.

\section{Limites}

Si le port du casque était différent chez les cyclistes qui n'ont pas participé à l'étude comparativement à l'échantillon choisi, un biais de sélection est possible. Malheureusement, outre l'absence d'information sur l'usage du casque chez ces patients, la nature du processus de collecte des données nous a empêchés de déterminer si les personnes que nous n'avons pas réussi à joindre ou qui ont refusé de participer à l'étude auraient été des cas ou des témoins. Si ceux qui ont refusé de participer étaient plus nombreux à porter leur casque incorrectement et ont par conséquent subi des blessures plus graves à la tête ou au visage, nous avons sousestimé l'effet protecteur d'un casque correctement ajusté ou qui demeure bien centré. Comme l'ajustement du casque était autodéclaré, une mauvaise classification est possible si les cyclistes étaient plus portés à indiquer que l'ajustement du casque était meilleur qu'il ne l'était en réalité. Il est possible que ceux n’ayant pas subi de blessure à la tête aient été plus nombreux à déclarer que l'ajustement du casque était excellent alors qu'il ne l'était pas nécessairement et que ceux ayant subi des blessures à la tête aient été moins nombreux à déclarer un ajustement excellent alors qu'il l'était peut-être en réalité. Si c'est le cas, l'effet d'un mauvais ajustement du casque a été surestimé. Lee et collab. ${ }^{9}$ ont observé que l'ajustement du casque perçu par les sujets était souvent surestimé comparativement à l'évaluation d'experts, ce qui signifie que les estimations du risque lié à l'ajustement $\mathrm{du}$ casque dans notre étude pourraient être biaisées. La correspondance observée entre l'ajustement du casque signalé initialement et celui déclaré au moment du suivi était élevée pour les cas $(91,7 \%)$ et les témoins $(85,5 \%)$, mais les valeurs kappa étaient basses pour les témoins et pourraient indiquer un biais de classification erronée faisant en sorte que les rapports de cotes se seraient faussement éloignés ou rapprochés de l'effet nul. Les estimations plus faibles de la fiabilité concernant la position du casque étaient similaires pour les cas et les témoins et pourraient indiquer une classification erronée qui aurait rapproché le rapport de cotes de l'effet nul.

Nous avons tenu compte de plusieurs facteurs de confusion possibles qui sont liés aux blessures à vélo : fréquence d'utilisation de la bicyclette, présence d'un compagnon, vitesse, IMC, sexe et âge. Dans leur étude, Rivara et collab. ${ }^{13}$ ont présenté des résultats non ajustés après avoir déterminé que la gravité de l'accident n'avait aucune influence sur les estimations de l'effet en ce qui concerne la relation entre le risque de blessures à la tête et l'ajustement ou la position du casque. Il est donc peu probable que d'autres facteurs liés tant aux blessures à la tête et au visage qu'à l'ajustement du casque puissent expliquer les effets que nous avons relevés.

\section{Conclusion}

L'ajustement et la position du casque pendant un accident peuvent modifier significativement le risque de blessures à la tête et au visage. Le port correct du casque peut être augmenté au moyen de programmes d'éducation informant les cyclistes qu'un casque ne peut pas assurer une protection complète s'il n'est pas bien ajusté. Les fabricants devraient poursuivre leurs efforts pour concevoir des casques de différentes formes et tailles qui sont faciles à utiliser et qui restent bien en place afin de mieux protéger les cyclistes. Les employés des commerces au détail qui vendent des casques doivent suivre une formation qui leur enseigne les principes $\mathrm{du}$ port correct du casque afin qu'ils puissent transmettre cette importante information aux consommateurs.

\section{Remerciements}

Les auteurs souhaitent remercier le personnel clinique et le personnel de recherche de l'Alberta Children's Hospital, du Foothills Medical Centre, du Rockyview General Hospital et du Peter Lougheed Centre de Calgary, de même que du Stollery Children's Hospital, de l'University of Alberta Hospital et du Northeast Community Health Centre d'Edmonton. Ils souhaitent remercier tout spécialement le personnel de recherche de l'étude et les étudiants d'Edmonton et de Calgary, la Paediatric Emergency Research Team (PERT), les bénévoles du Paediatric Emergency Medicine Research Associates Program (PEMRAP) de l'Alberta Children's Hospital et les assistants de recherche en médecine d'urgence du Foothills Medical Centre.

Le $\mathrm{D}^{\mathrm{r}}$ Brent Hagel est titulaire de l'Alberta Children's Hospital Foundation Professorship in Child Health and Wellness, financé par un donateur anonyme et par la Compagnie des chemins de fer nationaux du Canada. Il a aussi reçu le prix du chercheur en santé des populations de l'Alberta Heritage Foundation for Medical 
Research (AHFMR - maintenant l'Alberta Innovates-Health Solutions). Cette étude a été rendue possible grâce à la subvention d'établissement octroyée à $D^{r}$ Hagel par l'AHFMR. Le $\mathrm{D}^{\mathrm{r}}$ Rowe est titulaire de la Chaire de recherche du Canada en médecine d'urgence fondée sur les données probantes (niveau 1) des Instituts de recherche en santé du Canada (IRSC), qui est financée par le gouvernement du Canada. Les travaux de Jacqueline Williamson ont été financés par une bourse de stage de recherche d'été pour étudiants de l'O’Brien Centre for the Bachelor of Health Sciences program de l'Université de Calgary. Les travaux de Nicole Romanow ont été financés par une bourse de stagiaire de recherche du programme de formation des IRSC de l'Alberta Children's Hospital Research Institute.

\section{Références}

1. Thompson DC, Rivara FP, Thompson RS. Helmets for preventing head and facial injuries in bicyclists. Cochrane Database of Systematic Reviews. 1999;(4):CD001855.

2. Hagel BE, Rizkallah JW, Lamy A et collab. Bicycle helmet prevalence two years after the introduction of mandatory use legislation for under 18 year olds in Alberta, Canada. Inj Prev. 2006;12:262-5.

3. Swart R. The history of bicycle helmets. Arlington (VA) : Bicycle Helmet Safety Institute; 2010. Consultable en ligne à la page : http://www.helmets.org/history.htm

4. Ichikawa M, Nakahara S. School regulations governing bicycle helmet use and head injuries among Japanese junior high school students. Accid Anal Prev. 2007;39:469-74.

5. Robinson DL. Bicycle helmet legislation : can we reach a consensus? Accid Anal Prev. 2007;39:86-93.

6. Karkhaneh M, Rowe BH, Saunders LD, Voaklander DC, Hagel BE. Bicycle helmet use four years after the introduction of helmet legislation in Alberta, Canada. Accid Anal Prev. 2011;43:788-96.

7. Attewell RG, Glase K, McFaden M. Bicycle helmet efficacy : a meta-analysis. Accid Anal Prev. 2001;33:345-52.
8. Strohm PC, Sudkamp NP, Zwingmann J, El Saman A, Kostler W. Polytrauma in cyclists. Incidence, etiology, and injury patterns. Unfallchirurg. 2005;108:1022-4.

9. Lee RS, Hagel BE, Karkhaneh M, Rowe BH. A systematic review of correct bicycle helmet use : how varying definitions and study quality influence the results. Inj Prev. 2009;15:125-31.

10. Hagel BE, Lee RS, Karkhaneh M, Voaklander D, Rowe BH. Factors associated with incorrect bicycle helmet use. Inj Prev. 2010;16:178-84.

11. Hansen KS. Protective effect of different types of bicycle helmets. Traffic Inj Prev. 2003;4:285-90.

12. Amoros E, Chiron M, Martin JL, Thélot B, Laumon B. Bicycle helmet wearing and the risk of head, face, and neck injury: a French case-control study based on a road trauma registry. Inj Prev. 2012;18:27-32.

13. Rivara FP, Astley S, Clarren S, Thompson DC, Thompson RS. Fit of bicycle safety helmets and risk of head injuries in children. Inj Prev. 1999;5:195-7.

14. Rivara FP, Thompson DC, Thompson RS. Epidemiology of bicycle injuries and risk factors for serious injury. Inj Prev. 1997;3:110-4.

15. Wells S, Mullin B, Norton R et collab. Motorcycle rider conspicuity and crash related injury : case-control study. BMJ. 2004;328(7444):857-62.

16. Landis JR, Koch GG. The measurement of observer agreement for categorical data. Biometrics. 1977;33:159-74.

17. World Health Organization. BMI classification. Genève $(\mathrm{CH})$ : World Health Organization; 2006. Consultable en ligne à la page : http://apps.who.int/bmi/index.jsp?introPage = intro_3.html

18. Winters M, Davidson G, Kao D, Teschke K. Motivators and deterrents of bicycling : comparing influences on decisions to ride. Transportation. 2011;38:153-68.

19. Mickey RM, Greenland S. The impact of confounder selection criteria on effect estimation. Am J Epidemiol. 1989;129: 125-37.
20. White IR, Royston P, Wood AM. Multiple imputation using chained equations : issues and guidance for practice. Stat Med. 2010;30:377-99.

21. Foss RD, Beirness DJ. Bicycle helmet use in British Columbia : effects of the helmet use law. Chapel Hill (NC) : UNC Highway Safety Research Center, University of North Carolina; 2000. Joint publication of the Traffic Injury Research Foundation.

22. Page JL, Macpherson AK, MiddaughBonney T, Tator $\mathrm{CH}$. Prevalence of helmet use by users of bicycles, push scooters, inline skate and skateboards in Toronto and the surrounding area in the absence of comprehensive legislation : an observational study. Inj Prev. 2012;18:94-7. 